\title{
Effect of the Order of Test Diets on the Accuracy and Safety of Swallowing Studies
}

\author{
Se Hee Jung, MD, PhD, Juyong Kim, MD, Hyeonghui Jeong, MD, Shi-Uk Lee, MD, PhD \\ Department of Rehabilitation Medicine, Seoul National University College of Medicine, \\ Seoul National University Boramae Medical Center, Seoul, Korea
}

Objective To assess whether the order of test diets influences the results of swallowing studies with regard to their accuracy and safety.

Methods Subjects with suspected dysphagia underwent a videofluoroscopic swallowing study (VFSS) and/or a fiberoptic endoscopic evaluation of swallowing (FEES) and repeated the study on the same day or within a week. The order of test diets comprised of two different sets: trial 1 with the fluid first and trial 2 with the semi-solid food first. Main outcome measurements were the modified penetration-aspiration scale (mPAS) and the pharyngeal residue severity scale (PRSS) for the vallecula and the pyriform sinus.

Results Sixty-six patients (44 men and 22 women, aged 65.0 \pm 15.0 years) were enrolled in this study. Forty-three subjects were evaluated with VFSS only and 23 with both VFSS and FEES. As a result of the swallowing studies, there was no significant difference in each chosen diet sequence regarding mPAS and PRSS. Furthermore, there was no difference regarding the duration of studies, rate of premature study termination, rate of abnormal findings in post-study chest X-ray, and rate of fever or pneumonia post-study.

Conclusion The accuracy and safety of the swallowing studies do not rely on the order of test diets.

Keywords Deglutition disorders, Diet, Deglutition, Evaluation studies

\section{INTRODUCTION}

The swallowing function has been evaluated by direct observation of swallowing of various test diets, by studies such as videofluoroscopic swallowing study (VFSS), and

Received January 16, 2014; Accepted April 16, 2014

Corresponding author: Shi-Uk Lee

Department of Rehabilitation Medicine, Seoul National University College of Medicine, Seoul National University Boramae Medical Center, 20 Boramae-ro 5-gil, Dongjak-gu, Seoul 156-707, Korea

Tel: +82-2-870-2671, Fax: +82-2-870-3866, E-mail: shiuk.lee@gmail.com

(c) This is an open-access article distributed under the terms of the Creative Commons Attribution Non-Commercial License (http://creativecommons. org/licenses/by-nc/3.0) which permits unrestricted noncommercial use, distribution, and reproduction in any medium, provided the original work is properly cited.

Copyright $\odot 2014$ by Korean Academy of Rehabilitation Medicine by the fiberoptic endoscopic evaluation of swallowing (FEES) [1-6]. For these swallowing studies are various test diets with different rheologic properties used [7-12]. The risk of aspiration and the degree of pharyngeal residue of each diet are affected by the rheologic properties of the diets. Generally, test diets with a higher viscosity decrease the risk of aspiration but increase the pharyngeal residue [13-16].

During swallowing studies, the pharyngeal residue of the previous swallowing can affect the result of the subsequent test diet. Although the residue can be minimized by multiple swallowing, active cough, and suction, the residue of the previous diet may disrupt the accurate interpretation of the results of the swallowing study. In 
cases of massive aspiration during a study, the study may be earlier terminated for the safety of the patient and the rest of the test diets cannot be evaluated.

Therefore, starting the test with a test diet of higher viscosity (higher risk of pharyngeal residue) or with a diet of lower viscosity (higher probability of aspiration) may have different effects on the results of a study. However, there has been no evidence-based and standardized suggestion for the order of test diets yet.

Therefore, we aimed to assess whether the order of test diets influences the results of the swallowing studies with regard to their accuracy and safety.

\section{MATERIALS AND METHODS}

\section{Participants}

Any dysphagic patient aged 18 years and older was included who visited the Seoul National University Boramae Medical Center, Seoul, Korea. Exclusion criteria were as follows: history of an allergic reaction to lidocaine, iatrogenic alteration of pharyngeal anatomy, inability of the patient to maintain a sitting position for at least 20 minutes, inability to insert an endoscope into the nasal cavity, uncontrolled active nasal bleeding, pregnancy, breast-feeding and poor cooperation. Demographic data were obtained including age, sex, etiology, and days from onset to study. Before the swallowing study, the diets of patients were assessed by using the American SpeechLanguage-Hearing Association National Outcome Measurement System swallowing scale (ASHA NOMS) [17]. Clinical information, such as medical history and physical findings, were quantified by using a clinical dysphagia scale (CDS) $[3,18,19]$. This study was conducted in conformance with the ethical standards of the Declaration of Helsinki (1964) and was approved by the Institutional Review Board of our institution before study initiation. All participants gave their written informed consents before study inclusion.

\section{Food preparation}

Following 5 diets were used in this study: 1) thin fluid containing of $2 \mathrm{~mL} 35 \%$ diluted barium solution; 2) thickened fluid containing of $2 \mathrm{~mL}$ tomato juice-thick fluid (which was made by adding food thickener [Toromi UP; Nisshin Science Co., Yokohama, Japan] to 35\% diluted barium solution); 3 ) rice porridge containing of rice porridge coated with barium; 4) curd type yogurt containing of a mixture of commercial yogurt (Yoplait Classic; Binggrae Co., Seoul, Korea) and barium; and 5) boiled rice containing of boiled rice coated with barium. Every test diet was dyed with different colors to allow discrimination during FEES.

\section{Study protocol}

The order of test diets comprised two different sets: trial 1 and trial 2 . In trial 1 , the test diets were sequentially delivered in following order: thin fluid, thickened fluid, rice porridge, curd type yogurt, and boiled rice. In trial 2 , rice porridge, curd type yogurt, boiled rice, thickened fluid, and thin fluid were provided sequentially. Trials 1 and 2 were designed for the purpose to compare the effects regarding safety and accuracy. For that purpose, we designed trial 1 , beginning with thin fluid (a diet of lower possibility of pharyngeal residue with higher possibility of aspiration) and trial 2, beginning with rice porridge (a diet of higher possibility of pharyngeal residue with lower possibility of aspiration). Two swallows were evaluated for each test diet.

The dysphagic patients were randomly allocated to two groups (A and $\mathrm{B}$ ) by a random number generator. The study subjects underwent the VFSS and/or the FEES and they were simultaneously performed if the subject was evaluated by both studies. In that case, fiberoptic endoscope was inserted first; thereafter the food was

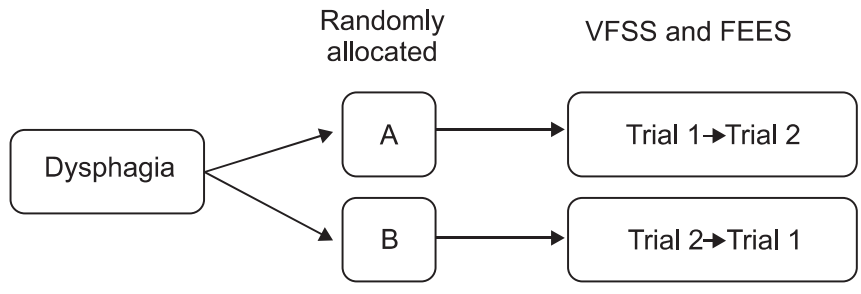

Trial 1: thin fluid $\rightarrow$ thickend fluid rice porridge $\rightarrow$ curd type yogurt $\rightarrow$ boiled rice

Trial 2: rice porridge $\rightarrow$ curd type yogurt $\rightarrow$ boiled rice $\rightarrow$ thickened fluid $\rightarrow$ thin fluid

Fig. 1. The patients were randomly allocated to two groups (A and B). Subjects underwent the swallowing studies and repeated the study on the same day or within a week. The order of test diets comprised of two different sets: trial 1 with the fluid first and trial 2 with the semisolid food first. Patients in group A underwent trial 1 first and trial 2 later. Patients in group B underwent trial 2 first and trial 1 later. VFSS, videofluoroscopic swallowing study; FEES, fiberoptic endoscopic evaluation of swallowing. 
swallowed with videofluoroscopic evaluation and at last followed an endoscopic evaluation.

A fluoroscope Medix 6000DR (Hitachi Medical Corporation, Tokyo, Japan) was used for VFSS. A transnasal endoscope (PENTAX Medical Company, Montvale, USA) and a video processor (PENTAX Medical Company) were used for FEES.

Patients in group A underwent trial 1 first and trial 2 later. Patients in group B underwent trial 2 first and trial 1 later. Swallowing studies with a different order of test diets were repeated on the same day or within a week. Videos were recorded and divided by test diets (Fig. 1).

The study was immediately stopped for safety reasons if there was massive aspiration (when most of the food bolus entered the airway). The study was also terminated if the patients showed a severe oral phase delay (food bolus stays in the mouth for more than 60 seconds without effective tongue motion) and the reason for the premature termination was recorded.

\section{Evaluation}

A physiatrist with more than 2 years experience in performing swallowing studies evaluated the VFSS and FEES. Also the order of test diets was blinded during video analysis. We used the modified penetration-aspiration scale (mPAS) to determine penetration and aspiration (Table 1). The PAS determines the severity of penetration and aspiration from level 1 to 8 depending on the depth

Table 1. The modified penetration-aspiration scale and the pharyngeal residue severity scale

\begin{tabular}{l} 
Modified penetration-aspiration scale \\
1. Material does not enter the airway \\
2. Material enters the airway, remains above the vocal \\
folds \\
3. Material enters the airway, contacts the vocal folds \\
4. Material enters the airway, passes below the vocal \\
folds and effort is made to eject \\
5. Material enters the airway, passes below the vocal \\
folds and no effort is made to eject \\
Pharyngeal residue severity scale \\
0. None \\
1. Coating: coating of the pharyngeal mucosa; no pooling \\
2. Mild: mild pooling/residue \\
3. Moderate: moderate pooling/residue \\
4. Severe: severe pooling/residue \\
\hline
\end{tabular}

that the test diet reached in the larynx and trachea and the elimination of the diet by the cough reflex [20]. The mPAS was revised from the PAS based on penetration, aspiration followed by cough reflex, and aspiration without cough reflex [21]. We also used the pharyngeal residue severity scale (PRSS) to evaluate the severity of residue in the vallecula and pyriform sinus [22] (Table 1).

Primary outcome variables of this study were as follows: differences in the mPAS and PRSS for the vallecula and the pyriform sinus of each diet between trials 1 and 2. We also recorded the total duration of each swallowing study for each patient, the number of studies which were terminated prematurely, any abnormal findings in the post-study chest X-ray and the presence of fever $\left(38.3^{\circ} \mathrm{C}\right.$ or higher) and pneumonia post-study (by clinical and laboratory findings within one week). All the outcome variables were evaluated by one physiatrist who was blinded to the information of subjects and diets and not involved in the swallowing studies.

The Wilcoxon signed-rank test was used to evaluate differences in outcome variables between trials 1 and 2 .

\section{RESULTS}

A total of 70 patients were asked to participate in this

Table 2. Demographic data of the participants

\begin{tabular}{lccc}
\hline \multicolumn{1}{c}{ Variable } & $\begin{array}{c}\text { Group A } \\
(\mathbf{n = 3 2})\end{array}$ & $\begin{array}{c}\text { Group B } \\
(\mathbf{n}=\mathbf{3 4})\end{array}$ & p-value \\
\hline Sex (male:female) & $21: 11$ & $23: 11$ & 0.86 \\
& $(65.6: 34.4)$ & $(67.6: 32.4)$ & \\
Age (yr) & $63.0 \pm 15.9$ & $66.9 \pm 14.0$ & 0.29 \\
Days from onset to study & $139 \pm 197$ & $232 \pm 728$ & 0.49 \\
Etiology & & & 0.06 \\
Ischemic stroke & $17(53.1)$ & $20(58.8)$ & \\
Hemorrhagic stroke & $4(12.5)$ & $8(23.5)$ & \\
Traumatic brain injury & $5(15.6)$ & $0(0.0)$ & \\
Brain tumor & $2(6.3)$ & $1(2.9)$ & \\
Parkinsonism & $0(0.0)$ & $3(8.8)$ & \\
Others & $4(12.5)$ & $2(5.9)$ & \\
ASHA NOMS & $3.3 \pm 2.5$ & $3.4 \pm 2.5$ & 0.87 \\
Clinical dysphagia scale & $25.5 \pm 21.5$ & $17.6 \pm 14.2$ & 0.10 \\
\hline
\end{tabular}

Values are presented as mean \pm standard deviation or number (\%).

ASHA NOMS, American Speech-Language-Hearing Association National Outcome Measurement System swallowing scale. 
study and 4 patients refused. A total of 66 subjects ( 44 men and 22 women, mean age $65.0 \pm 15.0$ years) were enrolled in the present study. Demographic data, ASHA NOMS and CDS scores showed no significant differences between the 2 groups (Table 2). Forty-three subjects were evaluated with the VFSS only and 23 with both the VFSS and FEES.

There was no significant difference in the MPAS and the PRSS in the vallecula and the pyriform sinus for each diet in trial 1 (which started with the thin fluid) and trial 2 (which started with the rice porridge, see Table 3) in VFSS or FEES (Table 4).

In group $\mathrm{A}$, two patients could not perform trial 2 because of massive aspiration during trial 1 , whereas in group $\mathrm{B}$, one patient could not perform trial 1 due to massive aspiration during trial 2 . Twenty out of 129 trials (15.5\%) failed to finish the VFSS because of severe oral phase delay in 8 trials (6.2\%) and due to massive aspiration in 12 trials $(9.3 \%)$. There was no significant difference in the rate of premature termination for both trials (Table 5).

Table 3. Differences in the results of the videofluoroscopic swallowing study between trials 1 and $2(n=66)$

\begin{tabular}{lccc}
\hline & Trial 1 & Trial 2 & p-value \\
\hline mPAS & & & \\
Thin fluid & $1.59 \pm 1.14$ & $1.73 \pm 1.30$ & 0.26 \\
Thickened fluid & $1.22 \pm 0.51$ & $1.33 \pm 0.66$ & 0.16 \\
Rice porridge & $1.27 \pm 0.84$ & $1.43 \pm 1.12$ & 0.19 \\
Curd type yogurt & $1.08 \pm 0.40$ & $1.08 \pm 0.40$ & 1.00 \\
\hline Boiled rice & $1.18 \pm 0.67$ & $1.22 \pm 0.71$ & 0.32 \\
\hline PRSS (vallecula) & & & \\
Thin fluid & $0.80 \pm 0.71$ & $0.90 \pm 0.71$ & 0.10 \\
\hline Thickened fluid & $0.96 \pm 0.58$ & $1.08 \pm 0.67$ & 0.17 \\
\hline Rice porridge & $1.20 \pm 0.79$ & $1.18 \pm 0.83$ & 0.80 \\
Curd type yogurt & $1.02 \pm 0.69$ & $0.98 \pm 0.72$ & 0.53 \\
Boiled rice & $1.29 \pm 0.96$ & $1.37 \pm 0.97$ & 0.31 \\
\hline PRSS (pyriform sinus) & & & \\
Thin fluid & $0.51 \pm 0.62$ & $0.57 \pm 0.65$ & 0.26 \\
\hline Thickened fluid & $0.57 \pm 0.61$ & $0.67 \pm 0.66$ & 0.06 \\
\hline Rice porridge & $0.82 \pm 0.63$ & $0.84 \pm 0.72$ & 0.74 \\
\hline Curd type yogurt & $0.67 \pm 0.63$ & $0.59 \pm 0.64$ & 0.16 \\
\hline Boiled rice & $0.76 \pm 0.75$ & $0.73 \pm 0.73$ & 0.80 \\
\hline
\end{tabular}

Values are presented as mean \pm standard deviation. mPAS, modified penetration-aspiration scale; PRSS, pharyngeal residue severity scale.
Furthermore, there was no difference in the duration of studies ( $489 \pm 353$ seconds in trial $1,517 \pm 348$ seconds in trial 2; $\mathrm{p}=0.82$ ), rate of abnormal findings in post-study chest X-ray ( 1 out of 65 in trial 1, 1 out of 64 in trial 2), rate of fever (none in both trials) and pneumonia post-study

Table 4. Differences in the results of fiberoptic endoscopic evaluation of swallowing between trials 1 and $2(n=23)$

\begin{tabular}{lccc}
\hline & Trial 1 & Trial 2 & p-value \\
\hline mPAS & & & \\
Thin fluid & $1.30 \pm 0.66$ & $1.30 \pm 0.66$ & 1.00 \\
Thickened fluid & $1.10 \pm 0.45$ & $1.10 \pm 0.45$ & 1.00 \\
Rice porridge & $1.05 \pm 0.22$ & $1.15 \pm 0.49$ & 0.32 \\
Curd type yogurt & $1.05 \pm 0.22$ & $1.05 \pm 0.22$ & 1.00 \\
Boiled rice & $1.00 \pm 0.00$ & $1.00 \pm 0.00$ & 1.00 \\
PRSS (vallecula) & & & \\
Thin fluid & $1.20 \pm 1.00$ & $1.25 \pm 1.02$ & 0.32 \\
Thickened fluid & $1.60 \pm 0.99$ & $1.45 \pm 1.05$ & 0.41 \\
\hline Rice porridge & $1.70 \pm 0.92$ & $1.70 \pm 0.92$ & 1.00 \\
Curd type yogurt & $1.70 \pm 1.22$ & $1.70 \pm 1.17$ & 1.00 \\
Boiled rice & $1.80 \pm 1.06$ & $1.90 \pm 1.07$ & 0.16 \\
PRSS (pyriform sinus) & & & \\
Thin fluid & $0.95 \pm 0.89$ & $1.00 \pm 0.86$ & 0.56 \\
\hline Thickened fluid & $1.25 \pm 0.91$ & $1.15 \pm 0.99$ & 0.41 \\
\hline Rice porridge & $1.30 \pm 0.98$ & $1.35 \pm 0.93$ & 0.56 \\
\hline Curd type yogurt & $1.30 \pm 1.34$ & $1.30 \pm 1.34$ & 1.00 \\
\hline Boiled rice & $1.20 \pm 1.15$ & $1.30 \pm 1.17$ & 0.16 \\
\hline
\end{tabular}

Values are presented as mean \pm standard deviation. mPAS, modified penetration-aspiration scale; PRSS, pharyngeal residue severity scale.

Table 5. Rate of videofluoroscopic swallowing study completion and causes of premature termination

\begin{tabular}{lccc}
\hline & $\begin{array}{c}\text { Trial 1 } \\
(\mathbf{n = 6 5 )}\end{array}$ & $\begin{array}{c}\text { Trial 2 } \\
(\mathbf{n = 6 4 )}\end{array}$ & $\begin{array}{c}\text { Total } \\
(\mathbf{n = 1 2 9})\end{array}$ \\
\hline $\begin{array}{l}\text { Complete study } \\
\text { Premature termination }\end{array}$ & $55(84.6)$ & $54(84.4)$ & $109(84.5)$ \\
$\quad \begin{array}{l}\text { Severe oral phase delay } \\
\text { Massive aspiration }\end{array}$ & $4(6.2)$ & $4(6.3)$ & $8(6.2)$ \\
\hline $\begin{array}{l}\text { Diets causing massive } \\
\text { aspiration }\end{array}$ & $6(9.2)$ & $6(9.4)$ & $12(9.3)$ \\
$\quad \begin{array}{l}\text { Thin fluid } \\
\text { Thickened fluid }\end{array}$ & 2 & 1 & 3 \\
\hline \multicolumn{1}{l}{ Rice porridge } & 1 & 1 & 2 \\
\hline
\end{tabular}

Values are presented as number (\%). 
(none in both trials).

\section{DISCUSSION}

The results of this study showed that the order of test diets did not significantly affect the evaluation of penetration, aspiration, and pharyngeal residues. Moreover, the order of test diets did not significantly change the total duration of study, the rate of prematurely terminated study, the rate of abnormal findings in post-study chest $\mathrm{X}$-ray as well as the rate of fever and pneumonia poststudy.

Several sequences of test diets have been proposed for a swallowing study. The order of consecutive diluted barium, pudding, and cookie was proposed by Logemann [12]. This method, which starts with fluid, has been widely used because liquids do not block the airway even if they are aspirated. Holas et al. [23] reported that pneumonia is less likely from aspiration of fluid than from aspiration of thicker foods $[12,24,25]$. There were several modified versions of the 'fluid-first' order of test diets [7$9,11]$. These methods considered safety issues first. However, although fluid has traditionally been thought to be safer than solid food when aspirated, fluid has a higher risk of aspiration than solid food $[12,14]$. Thus, some examiners prefer not to start with fluid $[10,21]$.

In this study, we tried two sets of test diets: one started with fluid and the other started with rice porridge. The main issue of this study was to compare between the start with fluid and the start with other than fluid. Although rice porridge had similar viscosity with curd type yogurt, we used both test diets for this study because they have different characteristics: the former need mastication and the latter do not. Moreover, boiled rice is used as the staple food in East Asian cultures. In Asia, rice porridge is frequently used as an alternative food for dysphagic patients due to its nature, easiness for mastication, lesser risk for aspiration than boiled rice, and cultural adaptability. Usually most of dysphagic patients in our hospital start dysphagic food with rice porridge and move on to boiled rice if possible. Therefore, above mentioned diets are used for the swallowing studies in our hospital and fit for the most of our dysphagic patients.

This study had some limitations. First, we did not discourage subjects from clearing the pharyngeal residue themselves by multiple swallowing or throat clearing.
These actions could have minimized the effect of pharyngeal residues of previous diets. Second, the swallowing function was not fully evaluated in patients who showed severe oral phase delay or massive aspiration during swallowing studies. Therefore, patients with severe dysphagia may have been excluded from the analysis. Thus, the findings of this study may not be applicable to patients with severe dysphagia. Third, most of our study subjects suffered from dysphagia secondary to neurologic etiologies. Therefore, the study findings may not be generalized to dysphagia of other etiologies, such as postoperative dysphagia. Fourth, most of the patients (58 of 66 patients) underwent trials 1 and 2 on the same day and the post-study chest X-ray was taken once after the completion of both studies. Therefore, a safety analysis could only be done for the 8 patients who underwent swallowing studies and post-study chest X-rays on two different days. Finally, only $23(34.8 \%)$ patients were evaluated by FEES because the remaining patients declined to perform the FEES or failed to cooperate during the examination. Thus, the statistical analysis was limited in the FEES.

In conclusion, the accuracy and safety of the swallowing studies did not rely on the order of the test diets. Thus, various orders of test diets can be chosen according to the characteristics of individual patients.

\section{CONFLICT OF INTREST}

No potential conflict of interest relevant to this article was reported.

\section{ACKNOWLEDGMENTS}

This study was supported by a grant of the Korea Health Technology R\&D Project, Ministry of Health \& Welfare, Korea (No. Al10691).

\section{REFERENCES}

1. Langmore SE, Schatz K, Olsen N. Fiberoptic endoscopic examination of swallowing safety: a new procedure. Dysphagia 1988;2:216-9.

2. Hiss SG, Postma GN. Fiberoptic endoscopic evaluation of swallowing. Laryngoscope 2003;113:1386-93.

3. Jung SH, Lee KJ, Hong JB, Han TR. Validation of clinical dysphagia scale: based on videofluoroscopic swal- 
lowing study. J Korean Acad Rehabil Med 2005;29:34350

4. Bingjie L, Tong Z, Xinting S, Jianmin X, Guijun J. Quantitative videofluoroscopic analysis of penetration-aspiration in post-stroke patients. Neurol India 2010;58:42-7.

5. Han TR, Paik NJ, Park JW. Quantifying swallowing function after stroke: a functional dysphagia scale based on videofluoroscopic studies. Arch Phys Med Rehabil 2001;82:677-82.

6. Paik NJ, Kim IS, Kim JH, Oh BM, Han TR. Clinical validity of the functional dysphagia scale based on videofluoroscopic swallowing study. J Korean Acad Rehabil Med 2005;29:43-9.

7. Mann G, Hankey GJ, Cameron D. Swallowing function after stroke: prognosis and prognostic factors at 6 months. Stroke 1999;30:744-8.

8. Ott DJ, Pikna LA. Clinical and videofluoroscopic evaluation of swallowing disorders. AJR Am J Roentgenol 1993;161:507-13.

9. Palmer JB, Kuhlemeier KV, Tippett DC, Lynch C. A protocol for the videofluorographic swallowing study. Dysphagia 1993;8:209-14.

10. Nishiwaki K, Tsuji T, Liu M, Hase K, Tanaka N, Fujiwara T. Identification of a simple screening tool for dysphagia in patients with stroke using factor analysis of multiple dysphagia variables. J Rehabil Med 2005;37:247-51.

11. Han TR, Paik NJ, Park JW, Kwon BS. The prediction of persistent dysphagia beyond six months after stroke. Dysphagia 2008;23:59-64.

12. Logemann JA. Evaluation and treatment of swallowing disorders. San Diego: College Hill Press; 1998.

13. Paik NJ, Han TR, Park JW, Lee EK, Park MS, Hwang IK. Categorization of dysphagia diets with the line spread test. Arch Phys Med Rehabil 2004;85:857-61.

14. Li M, Brasseur JG, Kern MK, Dodds WJ. Viscosity measurements of barium sulfate mixtures for use in motility studies of the pharynx and esophagus. Dysphagia
1992;7:17-30.

15. Clave P, de Kraa M, Arreola V, Girvent M, Farre R, Palomera E, et al. The effect of bolus viscosity on swallowing function in neurogenic dysphagia. Aliment Pharmacol Ther 2006;24:1385-94.

16. Goulding R, Bakheit AM. Evaluation of the benefits of monitoring fluid thickness in the dietary management of dysphagic stroke patients. Clin Rehabil 2000;14:11924.

17. American Speech-Language-Hearing Association; National Outcomes Measurement System. Adult speechlanguage pathology training manual. Rockville: American Speech-Language-Hearing Association; 1998.

18. Han TR, Paik NJ, Park JW. The clinical functional scale for dysphagia in stroke patients. Korean J Stroke 2001;3:153-7.

19. Chun SW, Lee SA, Jung IY, Beom J, Han TR, Oh BM. Inter-rater agreement for the clinical dysphagia scale. Ann Rehabil Med 2011;35:470-6.

20. Rosenbek JC, Robbins JA, Roecker EB, Coyle JL, Wood JL. A penetration-aspiration scale. Dysphagia 1996;11:93-8.

21. Kim JY, Koh ES, Kim HR, Chun SM, Lee SU, Jung SH. The diagnostic usefulness of the fiberoptic endoscopic evaluation of swallowing. J Korean Acad Rehabil Med 2011;35:14-22.

22. Kelly AM, Leslie P, Beale T, Payten C, Drinnan MJ. Fibreoptic endoscopic evaluation of swallowing and videofluoroscopy: does examination type influence perception of pharyngeal residue severity? Clin Otolaryngol 2006;31:425-32.

23. Holas MA, DePippo KL, Reding MJ. Aspiration and relative risk of medical complications following stroke. Arch Neurol 1994;51:1051-3.

24. Logemann JA. A manual for videofluoroscopic evaluation of swallowing. 2nd ed. Austin: Pro-Ed; 1993.

25. Langmore SE. Why I like the free water protocol? Perspect Swallowing Swallowing Disord (Dysphagia) 2011;20:116-20. 